\title{
Human umbilical cord-derived mesenchymal stem cells ameliorate insulin resistance by suppressing NLRP3 inflammasome-mediated inflammation in type 2 diabetes rats
}

Xiaoya Sun ${ }^{1}$, Haojie Hao², Qingwang Han², Xiaoyan Song ${ }^{2}$, Jiejie Liu², Liang Dong², Weidong Han ${ }^{2 *}$ (D and Yiming $\mathrm{Mu}^{1 *}$

\begin{abstract}
Background: Insulin resistance is one of the most common and important pathological features of type 2 diabetes (T2D). Recently, insulin resistance is increasingly considered to be associated with systemic chronic inflammation. Elevated levels of tumor necrosis factor (TNF)- $a$ and interleukin (IL)-1 $\beta$ in blood are predictive indicators of the development of T2D. Mesenchymal stem cell (MSC)-based therapies have been proven to have potential immunomodulation and anti-inflammatory properties through their paracrine effects; however, the mechanism for the anti-inflammatory effect of MSCs in enhancing insulin sensitivity is still uncertain.

Methods: In the present experiment, we used HepG2 cells, a human hepatoma cell line, and a MSC-HepG2 transwell culturing system to investigate the anti-inflammatory mechanism of human umbilical cord-derived MSCS (UC-MSCS) under palmitic acid (PA) and lipopolysaccharide (LPS)-induced insulin resistance in vitro. Insulin resistance was confirmed by glycogen assay kit and glucose assay kit. Inflammatory factor release was detected by ELISA, gene expression was tested by quantitative real-time PCR, and insulin signaling activation was determined by western blotting analysis. The changes of inflammatory factors and insulin signaling protein were also tested in T2D rats injected with UC-MSCs.

Results: Treating HepG2 cells with PA-LPS caused NLRP3 inflammation activation, including overexpression of NLRP3 and caspase- 1 , and overproduction of IL-1 $\beta$ and IL-18 as well as TNF- $a$ from HepG2 cells. The elevated levels of these inflammatory cytokines impaired insulin receptor action and thereby prevented downstream signaling pathways, exacerbating insulin resistance in HepG2 cells. Importantly, UC-MSCs cocultured with HepG2 could effectively alleviate PA and LPS-induced insulin resistance by blocking the NLRP3 inflammasome activation and inflammatory agents. Furthermore, knockdown of NLRP3 or IL-1 $\beta$ partially improved PA and LPS-induced insulin signaling impairments in the presence of UC-MSCs. Similarly, UC-MSC infusion significantly ameliorated hyperglycemia in T2D rats and decreased inflammatory activity, which resulted in improved insulin sensitivity in insulin target tissues.

(Continued on next page)
\end{abstract}

\footnotetext{
* Correspondence: hanwdrsw69@yahoo.com; muyiming@301hospital.com.cn

${ }^{2}$ Institute of Basic Medicine Science, College of Life Science, Chinese PLA General Hospital, Beijing 100853, China

'Department of Endocrinology, Chinese PLA General Hospital, Beijing 100853, China
} 
(Continued from previous page)

Conclusions: Our results indicated that UC-MSCs could attenuate insulin resistance and this regulation was correlated with their anti-inflammatory activity. Thus, MSCs might become a novel therapeutic strategy for insulin resistance and T2D in the near future.

Keywords: Mesenchymal stem cells, Inflammation, Insulin resistance, NLRP3 inflammasome, Palmitic acid, Lipopolysaccharide, Type 2 diabetes

\section{Background}

Pronounced changes in lifestyle and environment have made type 2 diabetes (T2D) a worldwide epidemic rapidly over the twenty-first century, and the accompanying complications constitute a main threat to global health [1]. Insulin resistance, a hallmark of T2D, is believed to be a fundamental pathologic event and underlying feature of T2D [2]. Although conventional insulin sensitizers, including metformin and rosiglitazone, have been proven to improve insulin sensitivity in target tissues, no pharmacologic agents exist which can be proven to treat diabetes completely. Hence, more efficacious strategies that can act via a new mechanism to promote insulin sensitivity are needed. Recently, insulin resistance is increasingly considered to be associated with low-grade systemic chronic inflammation, which has a central role in the pathogenesis of T2D [3, 4]. Since Hotamisligil et al. [5] first found that tumor necrosis factor (TNF)- $\alpha$ can be induced in a T2D rodent model, more attention has been focused on the connection between inflammation and insulin resistance. Another study in individuals revealed that, during obesity, elevated levels of $\mathrm{C}$ reactive protein and interleukin (IL)-1 $\beta$ in the blood were predictive indicators of the development of T2D [6]. Therefore, chronic inflammation has been recognized as a critical inducer in the development of insulin resistance and T2D.

Consistent with these data, recent studies have shown that the nod-like receptor protein 3 (NLRP3) inflammasome plays a pivotal regulatory role in the mechanism that induces systemic inflammation and insulin resistance in obesity and T2D $[7,8]$. The NLRP3 inflammasome can be triggered by both pathogen-associated molecular patterns and various danger-associated molecular patterns, including lipopolysaccharide (LPS) and saturated fatty acids, and further binds to its receptor on the cell surface, activating a proinflammatory pathway and inducing cytokine expression in various cell types $[9,10]$. Structurally, a functional NLRP3 inflammasome is composed of NLRP3, apoptosisassociated speck-like protein (ASC), and caspase-1. NLRP3 interacts with ASC to activate caspase-1 and further regulates the maturation and secretion of proinflammatory cytokines IL-1 $\beta$ and IL-18, which are involved in the inflammation response $[11,12]$. Indeed, multiple studies have demonstrated that inflammasome activation and the cleavage of inflammatory cytokines IL-1 $\beta$ and IL18 induced by obesity in key metabolic tissues promote chronic inflammation and contribute to the development of T2D [7, 13-15]. Other researchers reported that the elevated cytokines such as caspase- 1 , IL- $1 \beta$, IL- 6 , and TNF- $\alpha$ produced by activation of the inflammatory signaling pathways can contribute to glucose uptake failure and insulin sensitivity by disrupting insulin signaling [16-18]. Moreover, deficiency of protein in the NLRP3 inflammasome complex protects mice from high fat diet (HFD)-induced inflammation, alleviates insulin resistance, and promotes insulin signaling in insulin target tissues $[8,19,20]$. Notably, searching for an effective method to attenuate NLRP3 inflammasome-mediated inflammation will be a novel advance in treatment for insulin resistance and T2D.

Mesenchymal stem cells (MSCs) are multipotent stem cells with self-renewing capacities and low immunogenicity, which make them attractive for treating many diseases [21]. Interestingly, a recent paradigm shift suggests that MSCs have exhibited potential immunomodulation and anti-inflammatory properties through their paracrine effects [22]. In a renal medullary inflammation rat model, MSC transplantation could attenuate activation of the NLRP3 inflammasome and promote renal medullary function [23]. Moreover, MSCs have also been shown to downmodulate the inflammatory factors (IL- $1 \beta$, TNF- $\alpha$, and IL-6) through secreting prostaglandin $E_{2}$ for therapies in osteoarticular diseases [24]. Additionally, promising results in a clinical trial have shown that MSCs reduce systemic inflammation in patients with T2D, and our previous study has confirmed that MSC administration alleviates insulin resistance in target tissues of HFD-treated T2D rats $[25,26]$. Given the beneficial anti-inflammatory property of MSCs, it is imperative to test the possibility that MSCs could suppress NLRP3 inflammation activity to improve insulin resistance in a paracrine fashion.

In this study, we confirmed in vitro that the NLRP3 inflammasome was activated in an LPS and palmitic acid (PA)-induced inflammation model of insulin resistance in HepG2 cells. Human umbilical cordderived MSCs (UC-MSCs) and their conditioned media (CM) could enhance insulin sensitivity through inhibiting the upregulation of NLRP3 inflammasome components with the elevated cleavage of IL- $1 \beta$, IL18 , and TNF- $\alpha$ in insulin-resistant HepG2 cells. We 
also demonstrated that UC-MSCs repaired the glucose intolerance by suppressing inflammatory mediator release in insulin target tissues of the T2D animal model. Collectively, our studies provide evidence that MSC-mediated paracrine properties exert a protective effect on ameliorating insulin resistance through their immunomodulatory potency. This research further provides a rationale for the possible application of MSCs in the clinical treatment of insulin resistance and T2D.

\section{Methods}

\section{Cells and cell culture}

The UC-MSCs were freshly isolated from UCs of women after deliveries in the Chinese PLA General Hospital. Fibroblasts used for the experiment were obtained from cryopreserved cells from the dermis of healthy adults. UC-MSCs were isolated, amplified, and identified to meet the characteristics of MSCs using methods described previously [27, 28]. UC-MSCs were seeded and made adherent in serum-free Dulbecco's modified Eagle's medium (DMEM)/F12 medium (HyClone) supplemented with $10 \%$ fetal bovine serum (FBS; Hyclone) and $1 \times$ antibiotic/antimycotic (all from Invitrogen) overnight. When UC-MSCs were laid in passages 2 and 4, fresh media were collected after $48 \mathrm{~h}$ and centrifuged at $1000 \mathrm{rpm}$ for 5 minutes with low temperature to obtain $\mathrm{CM}$. The CM were then concentrated to 20 times through Vivaspin 20 (cutoff of $3 \mathrm{kDa}$; GE Healthcare UK Ltd, UK), and the concentrated CM were further filtered through a $0.22-\mu \mathrm{m}$ syringe filter in a sterile environment and stored at $-80{ }^{\circ} \mathrm{C}$. The serum-free culture medium was concentrated as a negative control.

HepG2 cells (from ATCC) were cultured in DMEM (low glucose; Invitrogen) at $37{ }^{\circ} \mathrm{C}$ in a $5 \% \mathrm{CO}_{2}$ atmosphere. Following starvation with serum-free DMEM containing $0.5 \%$ bovine serum albumin (BSA; Nanjing Sunshine Biotechnology Co. Ltd, China) for 16 h, $2 \times 10^{5}$ HepG2 cells were pretreated with $10 \mu \mathrm{g} / \mathrm{ml}$ LPS (Sigma) and then $0.25 \mathrm{mM}$ PA (Sigma) added that conjugated to fatty-acid free BSA (Sigma) for $24 \mathrm{~h}$, and were then incubated with either MSC-CM or cocultured with $1 \times 10^{5}$ UC-MSCs using transwell plants for another $24 \mathrm{~h}$. A third group, which served as a background control for UC-MSCs, was cocultured with $1 \times 10^{5}$ fibroblasts. Finally, cells were washed twice with polybutylene succinate (PBS; Sigma) and stimulated with $100 \mathrm{nM}$ insulin (Sigma) during the last $3 \mathrm{~h}$. Third-passage UC-MSCs were used for all of the coculturing experiments in this study.

\section{Animal experiment}

Male Sprague-Dawley (SD) rats 8 weeks old were obtained from the Chinese PLA General Hospital and housed at a constant temperature $\left(23 \pm 1{ }^{\circ} \mathrm{C}\right)$ with a 12 -h light and dark cycle and were allowed free access to water. For the HFD/STZ-induced T2D model, rats were given a HFD diet (40\% fat, $41 \%$ carbohydrate, and $19 \%$ protein) for 8 weeks, followed by intraperitoneal injection of streptozotocin (STZ) (25 mg/kg; Sigma-Aldrich) as described previously [29]. Control rats were fed an 8week regular chow diet. One week after STZ injection, we performed the intraperitoneal glucose tolerance tests (IPGTTs) and intraperitoneal insulin tolerance tests (IPITTs) to ensure the T2D model. At the same time, UC-MSC infusions $\left(3 \times 10^{6}\right.$ MSCs suspended in $0.5 \mathrm{ml}$ PBS) were administered to T2D rats via the tail vein. T2D rats receiving $0.5 \mathrm{ml}$ PBS were the control group. To judge the effect of UC-MSCs, blood glucose, IPGTTs, IPITTs, the levels of serum insulin, and Cpeptide were measured again at the appointed time.

\section{Glycogen content assay}

The glycogen content of cells was measured by glycogen assay kit (Sigma-Aldrich). The blue compound generated by the reaction was assayed at $620 \mathrm{~nm}$. The protein content of the collected HepG2 cells was quantified with the Bicinchoninic Acid (BCA) Protein Assay kit (Nanjing Bai Si Kai Co., China). Values were presented as the ratio of glycogen/protein (milligrams per grams).

\section{Glucose utilization assay}

HepG2 cells were seeded and then treated with LPS, PA, and UC-MSCs at different times. Plates with medium containing 10\% FBS were the control group. Glucose content in the media was assayed with a glucose assay kit (Sigma). Glucose-uptake content was obtained by the control group minus the experimental group. The glucose concentration was also normalized with the cellular protein concentration.

\section{Intraperitoneal glucose tolerance test and insulin tolerance test}

The IPGTTs and IPITTs were performed on overnight fasted rats through injection with $2 \mathrm{~g} / \mathrm{kg}$ glucose or $1 \mathrm{U}$ insulin/kg intraperitoneally. At the specified time, the glucose concentrations were achieved via tail-vein blood samples. According to the area under the curve, we assessed the available-use ratio of insulin in SD rats to determine the extent of insulin resistance.

\section{Enzyme-linked immunosorbent assay}

The concentrations of IL- $1 \beta$, IL-18, and TNF- $\alpha$, and serum insulin and serum $C$-peptide levels, were determined with specific ELISA kits (R\&D Systems, Minneapolis, MN, USA). 


\section{Quantitative real-time PCR}

We measured the gene expression of inflammatory factors (NLRP3, IL-1 $\beta$, IL-18, and TNF- $\alpha$ ) with quantitative real-time PCR (qRT-PCR). Trizol reagent (Invitrogen) was used to isolate the RNA and further synthesize single strands of cDNA using a mix of oligo(dT) and random primers with the Superscript RT Kit (all from Thermo Fisher Scientific, Waltham, MA, USA) according to the manufacturer's instructions. By following the appropriate amplification conditions for each set of primers, NLRP3, IL-1 $\beta$, IL-18, and TNF- $\alpha$ measurements were performed on a 7,500 Fast Real-Time PCR instrument (Applied Biosystems, Foster City, CA, USA). $\beta$ actin was used as the reference gene.

\section{Caspase- 1 activity assay}

HepG2 cells in all groups were scraped in cell lysis buffer; for all groups, reaction buffer and YVAD-AFC substrate were further added to measure the activation of caspase- 1 according to the instructions of a commercially available caspase-1 activity assay kit (Abcam, Cambridge, UK).

\section{Western blot analysis}

Sample protein concentrations of cells or tissues were determined by BCA Protein Assay kit. Typically, total protein $(40 \mu \mathrm{g})$ was electrophoresed by $8 \%$ SDS-PAGE and then transferred onto PVDF membranes. Primary antibodies used in this study were anti-NLRP3, anti-IRS (for phosphor-Ser ${ }^{307}$ IRS), anti-IRS, anti-PI3K, anti-AKT (for phosphor-Thr ${ }^{308} \mathrm{AKT}$ ), anti-AKT, and anti-Glut4 (all from Cell Signaling Technology, Danvers, MA, USA). After blocking, membranes were immunoblotted with primary and secondary antibodies, followed by detection with an ECL system.

\section{Lentiviral vectors construction and shRNA transfection to HepG2 cells}

For shRNA experiments, shRNA and lentivirus were constructed by our laboratory. The sequences of NLRP3 and IL-1 $\beta$ are presented in Additional file 1: Figure S1. GFP lentiviral vectors were produced by transfection of $293 \mathrm{~T}$ cells and the viral titers reached $2 \times 10^{9} \mathrm{TU} / \mathrm{ml}$ for further studies. The HepG2 cells were transfected with lentiviruses for NLRP3 and IL-1 $\beta$. The medium was replaced $12 \mathrm{~h}$ later, and these shRNA HepG2 cells were incubated with PA-LPS for another $24 \mathrm{~h}$. To identify the effect of RNA interference, the NLRP3 expression in the transfected cells treated with NLRP3-shRNA lentivirus or control-shRNA lentivirus and nontransfected HepG2 cells were detected by qRT-PCR and western blotting analysis. For IL-1 $\beta$-shRNA, the gene expression and protein level of IL-1 $\beta$ were quantified among these groups with the same methods.

\section{Statistical analysis}

We use Student's $t$ test to determine significant differences between two groups and one-way analysis of variance (ANOVA) to compare mean values with more than two groups. Values of $P<0.05, P<0.01$, and $P<0.001$ were considered statistically significant. All data are represented as mean \pm standard deviation.

\section{Results}

PA-LPS-stimulated inflammatory cytokine release in insulin-resistant HepG2 cells

PA-BSA combined with LPS treatment is known to be a common insulin-resistance inducer, so we choose PA-BSA plus LPS (PA-LPS) to stimulate HepG2 cells and induce the insulin-resistance model. PA-LPS treatment significantly decreased the glycogen content compared with the blank group, although the PA-BSA or LPS-alone group also showed partially impaired glycogen synthesis (Fig. 1a). Moreover, success of the insulin resistance model was further confirmed by an obvious reduction in insulinstimulated glucose uptake shown in the PA-LPS group (Fig. 1b). Next, we investigated the effect of PA-LPS on the production of inflammatory cytokines. By and large, PALPS evoked substantial IL-1 $\beta$ release with low production in either the PA-BSA or LPS-alone group (Fig. 1c). The secretions of IL-18 and TNF- $\alpha$ were also increased the most in HepG2 cells treated with PA-LPS complex (Fig. 1d, e). These results indicate that PA-LPS could induce expression of inflammatory mediators in insulin resistance of HepG2 cells.

\section{UC-MSCs attenuated inflammation and improved insulin sensitivity in vitro}

To investigate the anti-inflammatory effect of UC-MSCs, the PA-LPS-treated HepG2 cells were further cocultured with UC-MSCs in a transwell system. The fibroblasts were used as the control. To identify the characteristics of UCMSCs, morphology (Additional file 2: Figure S2a), adipocytic and osteoblastic differentiation capacity (Additional file 2: Figure S2b, c), and phenotypes (Additional file 2: Figure S2d) were analyzed. Analyzing genes encoding inflammatory molecules (NLRP3, IL-1 $\beta$, IL-18, and TNF- $\alpha$ ), RNA expression revealed lower expression in the UCMSC-treated group compared with the PA-LPS group (Fig. 2a-d). As recent studies reported that secretomes from stem cells had considerable anti-inflammatory potential [30-32], we also administered the CM into the insulin-resistant HepG2 cell and observed significant differences for the release of IL-1 $\beta$, IL-18, and TNF- $\alpha$ either in UC-MSCs or in the CM group compared to the PALPS group (Fig. 2e-g). The results revealed that MSC treatment had lower inflammatory factor production than MSC-CM. Consistently, the MSC group had lower caspase-1 activity of a 1.4-fold increase, with a 2.3-fold 
a

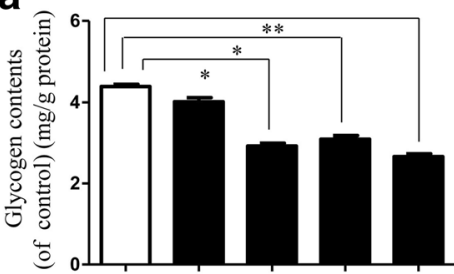

LPS $-\quad-\quad+\quad+\quad+$

$\begin{array}{rrrrrr}\mathrm{PP} & - & - & + & - & + \\ \mathrm{BSA} & - & + & + & - & +\end{array}$ b

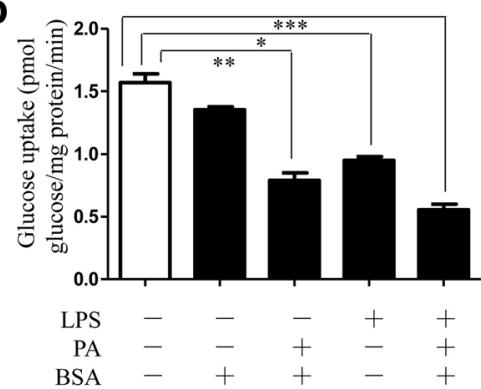

C

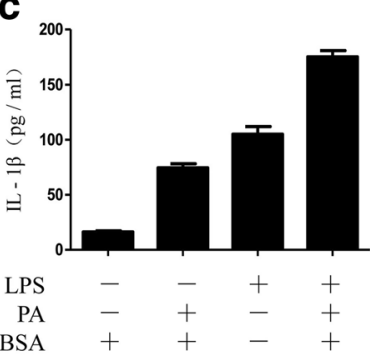

d

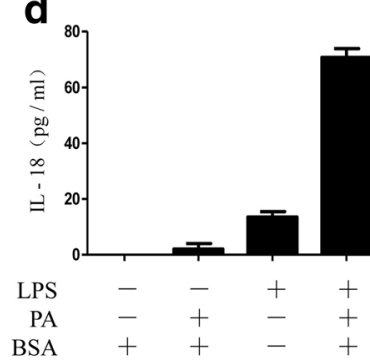

e

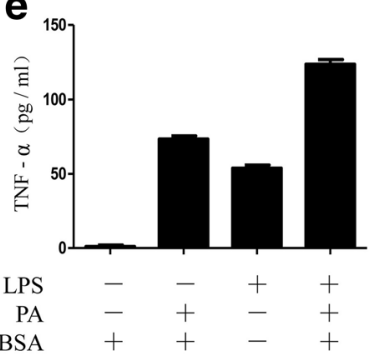

Fig. 1 PA-LPS stimulated inflammatory cytokine release in insulin-resistant HepG2 cells. HepG2 cells were incubated with BSA, PA-loaded BSA, LPS, and PA-LPS and then stimulated with insulin (100 nm) for $3 \mathrm{~h}$. (a) Glycogen content in cells determined using a glycogen assay kit. (b) A quantity of $300 \mu \mathrm{l}$ media in all groups was measured before and after the inducer by glucose assay kit, whose difference values were further normalized with cellular protein concentration. (c-e) Levels of IL-1 $\beta, I L-18$, and TNF-a quantified by a commercial ELISA kit. * $P<0.05$, ** $P<0.01$, ${ }^{* * *} P<0.001$. BSA bovine serum albumin, IL interleukin, LPS lipopolysaccharide, PA palmitic acid, TNF tumor necrosis factor

increase in the CM group, whereas the activity of caspase1 in PA-LPS cells was almost 4.8-fold compared to the blank group (Fig. $2 \mathrm{~h}$ ). These results revealed that MSCs could exert an anti-inflammatory effect partially via their paracrine role.

To further observe the effect of UC-MSCs on insulin resistance, we measured both glycogen synthesis and glucose uptake which were significantly increased in PA-LPS-treated HepG2 cells (Fig. 2i, j). Additionally, levels of NLRP3 and p-Ser ${ }^{307}$ IRS were reduced, while proteins like PI3K and phosphorylation of AKT were elevated and Glut4 translocation was also upregulated by UC-MSC treatment (Fig. $2 \mathrm{k}$ ). Together, these results indicate that MSCs alleviated inflammation and promoted glucose utilization by promoting an insulin signaling pathway in HepG2 cells.

\section{Effect of LY294002 on PI3K signaling and inflammation}

To further explore the association between inflammation and the insulin signaling pathway, LY294002, a specific inhibitor of PI3K, was given $1 \mathrm{~h}$ before adding PA-LPS. In the pretreatment of LY294002, the gene expression of NLRP3, IL-1 $\beta$, IL-18, and TNF- $\alpha$ was upregulated in insulin-resistant cells, which were reduced through coculturing with MSCs (Fig. 3a-d). Similarly, ELISA analysis of the supernatant showed the production of inflammatory mediators (IL- $1 \beta$, IL-18, and TNF- $\alpha$ ) was increased after PA + LPS treatment in the LY294002 group, which was significantly decreased after MSC interference (Fig. 3e-g). A similar result was obtained in caspase- 1 activity assay (Fig. $3 \mathrm{~h}$ ). Western blot analysis revealed downregulation of NLRP3 proteins by UC-MSCs, whereas decreased expression of PI3K, p-Thr ${ }^{308} \mathrm{AKT}$, and Glut4 in insulin-resistant cells was not reversed by MSCs after LY294002 treatment (Fig. 3i). Hence, these data further demonstrate that UC-MSCs could attenuate inflammation without the influence of LY294002 and were able to enhance insulin sensitivity through upregulating PI3K-Akt signaling transduction.

\section{UC-MSCs alleviated insulin resistance in $\mathrm{NLRP}^{-/-}$and IL- $1 \beta^{-/-}$HepG2 cells}

Next, we studied whether the NLRP3 inflammasomedependent IL-1 $\beta$ production contributed to insulin resistance. HepG2 cells were transfected with shRNA for NLRP3 and IL-1 $\beta$ (NLRP3 ${ }^{-/-}$cells and IL-1 $\beta^{-/-}$cells) (Additional file 3: Figure S3a, b). The results of both RT-PCR and ELISA analysis revealed that in the presence of PA-LPS, the mRNA expression and secretion of inflammatory factors were significantly lower in $\mathrm{NLRP}^{-/-}$cells compared with the WT HepG2 cells, while no significant change was seen by culturing with MSCs (Fig. $4 \mathrm{a}-\mathrm{g}$ ). A similar result was confirmed by caspase- 1 activity (Fig. $4 \mathrm{~h}$ ). We next examined the effect of NLRP3 on insulin receptor signaling, and protein analysis showed that $\mathrm{NLRP}^{-/-}$cells had higher insulin signaling (Fig. 4i). 


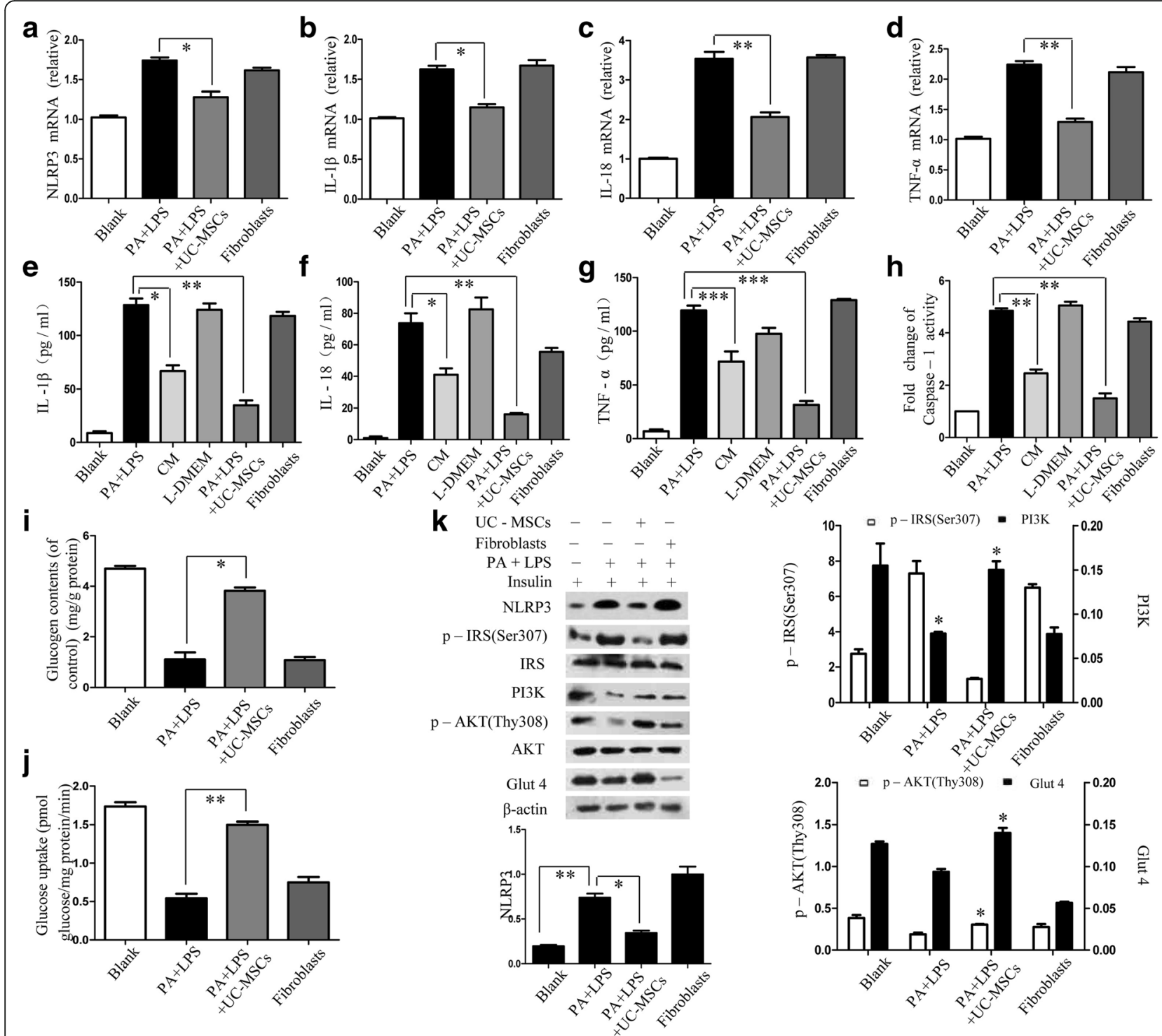

Fig. 2 UC-MSCS attenuated PA-LPS-induced inflammation and improved insulin sensitivity in vitro. HepG2 cells were treated with PA+ LPS, CM, L-DMEM, UC-MSCS, and fibroblasts accordingly. (a-d) mRNA of NLRP3, IL-1 $\beta$, IL-18, and TNF-a analyzed by quantitative RT-PCR. Values shown as fold change to the blank control. Protein levels were detected in HepG2 cells by ELISA for IL-1 $\beta$ (e), IL-18 (f), and TNF- $a$ (g). (h) Activity of caspase-1 assayed for each group. $(\mathbf{i}, \mathbf{j})$ Glucose metabolism of HepG2 cells in different groups by glycogen assay kit or glucose assay kit. (k) Western blot analysis of NLRP3, p-IRS (Ser307), IRS, PI3K, p-AKT (Thr308), AKT, and Glut4 of each group. Ratios of NLRP3, PI3K, Glut4 to $\beta$-actin, p-IRS to IRS, and $p$-AKT to AKT were quantitated. ${ }^{*} P<0.05,{ }^{*} P<0.01$. IL interleukin, LPS lipopolysaccharide, PA palmitic acid, TNF tumor necrosis factor, UC-MSC umbilical cord-derived mesenchymal stem cell

The results show that the insulin resistance was partially attributable to the NLRP3 inflammasome.

To better understand the role of NLRP3-dependent IL- $1 \beta$, we administered PA-LPS to IL- $1 \beta^{-/-}$cells and further cultured them with UC-MSCs. The transcription and secretion of IL- $1 \beta$ were reduced after PA-LPS stimulation. However, the levels of Nlrp3, IL-18, and TNF- $\alpha$ in IL- $1 \beta^{-/-}$cells were increased by adding PALPS compared to the IL-1 $\beta^{-1-}$ control cells. Additionally, these effects were reversed by coculturing with UC-
MSCs (Fig. 5a-g). The activity of caspase- 1 was elevated after PA-LPS treatment and then reduced through culturing with UC-MSCs (Fig. $5 \mathrm{~h}$ ). Subsequently, we assessed the effect of IL- $1 \beta$ on insulin signaling. Protein analysis showed lower expression of p-IRS, and elevated expression of PI3K, p-Akt, and GLUT4 in IL-1 $\beta^{-/-}$cells with PA-LPS treatment (Fig. 5i). This effect was not changed by culturing with UC-MSCs. All of the data suggested that IL-1 $\beta$ negatively regulated insulin downstream signaling. 


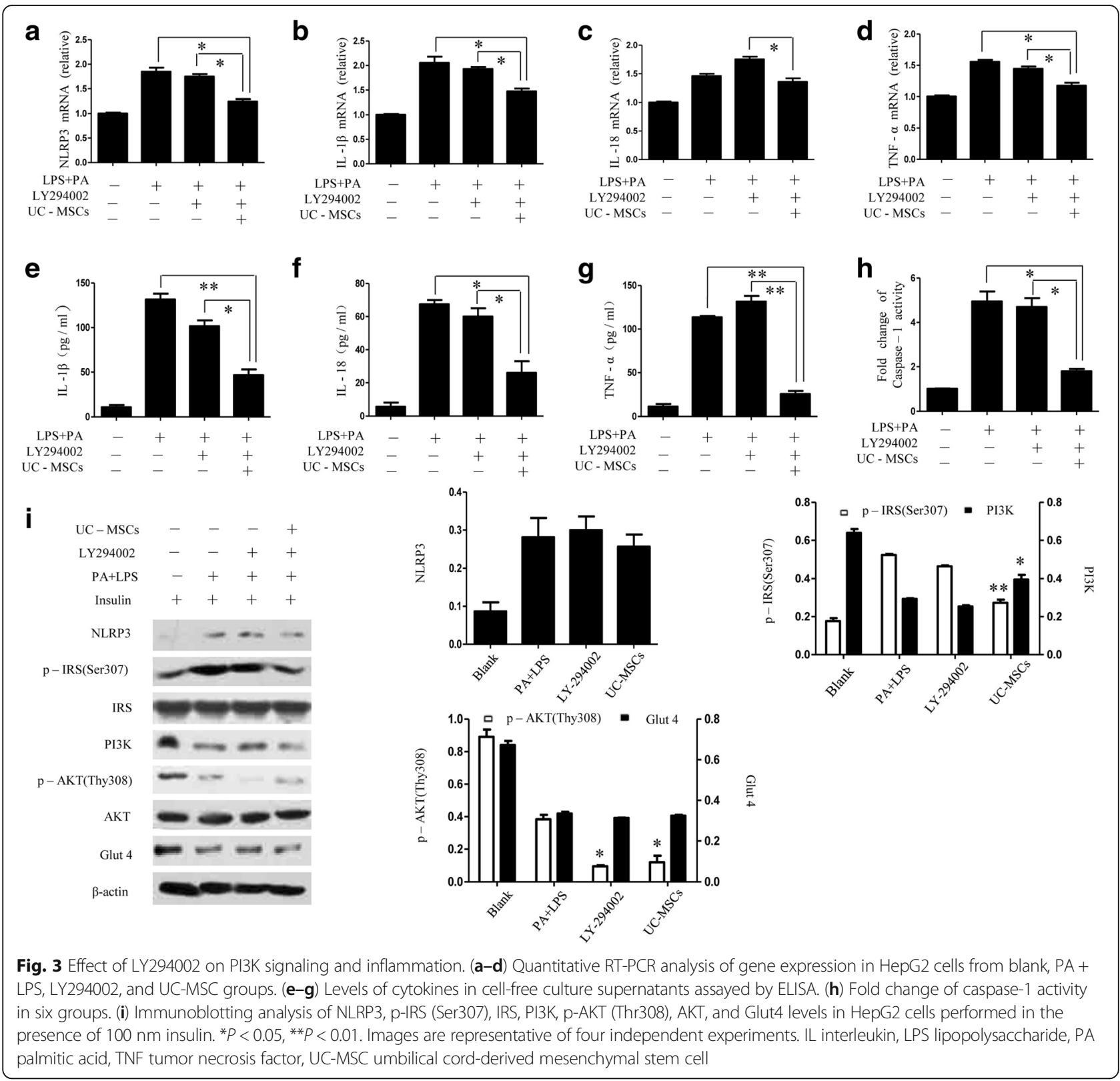

Taken together, these results demonstrate that NLRP3 impaired the normal insulin signaling pathway and led to insulin resistance; IL-1 $\beta$, a downstream molecular of NLRP3, also played a key role in regulating insulin sensitivity. UC-MSCs could further reduce these inflammatory parameters for increasing insulin sensitivity.

\section{UC-MSC infusion improved hyperglycemia and insulin sensitivity in $\mathrm{T} 2 \mathrm{D}$ rats}

To test the hypoglycemic effect of UC-MSCs in vivo, we investigated SD rats with $\mathrm{HFD} / \mathrm{STZ}$ to induce an insulin resistance model that further received UC-MSC infusion via the tail vein. The significant weight gain (data not shown) and elevation in blood glucose confirmed the success of the T2D model. From day 7 onward after UCMSC injection, the MSC-treated rats showed an obvious decrease in blood glucose compared with a consistent hyperglycemia in untreated T2D rats, although we observed a gradual increase of blood glucose level during our study period (Fig. 6a). Moreover, the IPGTT was performed, and the results showed that UC-MSCs contributed to a significant improvement of glucose tolerance compared to the T2D group (Fig. 6b). Accordingly, insulin sensitivity was also alleviated greatly in the MSC-treated group (Fig. 6c). Moreover, serum insulin concentration declined markedly in T2D rats and MSC treatment could induce a significant increase in insulin concentration (Additional file 4: Figure S4a). The 


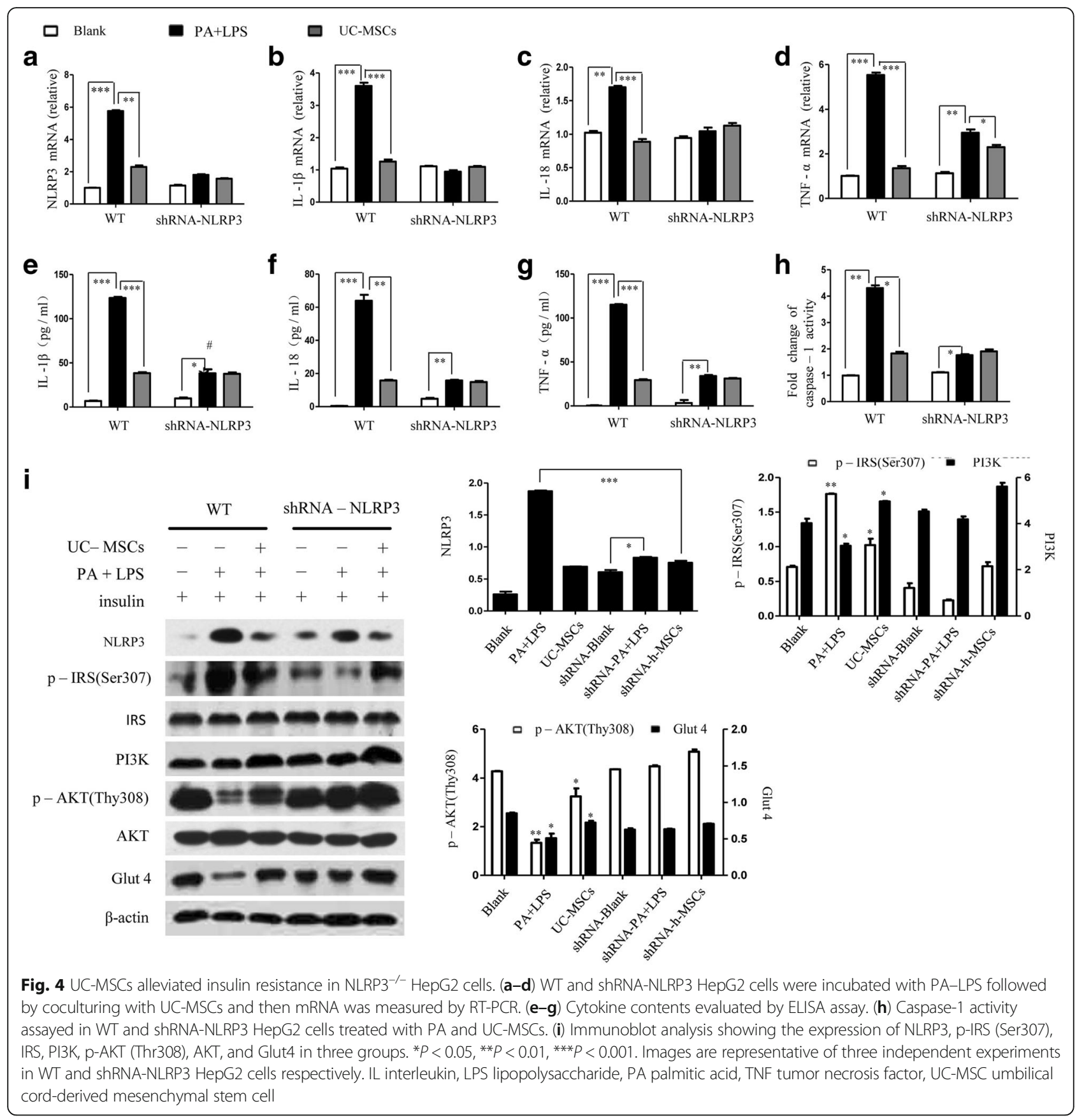

variation trend of serum $\mathrm{C}$-peptide was similar to that of insulin concentration in our study (Additional file 4: Figure S4b). These results demonstrate that MSC administration contributed to amelioration of hyperglycemia and insulin resistance in $\mathrm{T} 2 \mathrm{D}$ rats.

\section{UC-MSCs attenuated insulin resistance and inflammation} in target tissues

To further address whether UC-MSCs reveal therapeutic abilities to attenuate insulin resistance in vitro, we measured the inflammatory factor production and insulin signaling transduction in liver, adipose, and skeletal muscle tissues. We particularly studied the liver because it preceded peripheral insulin resistance during T2D development. The expression of genes (NLRP3, IL-1 $\beta$, IL-18, and TNF- $\alpha$ ) in the liver was dramatically increased by HFD plus STZ; however, all genes showed restoration by MSC interference (Fig. 7a). ELISA analysis results from liver tissue also showed decreased IL-1 $\beta$, IL-18, and TNF- $\alpha$ release after MSC treatment (Fig. 7b). Then, we continued to determine the expression of insulin signaling proteins by western blot analysis. p-Ser ${ }^{307}$ IRS 


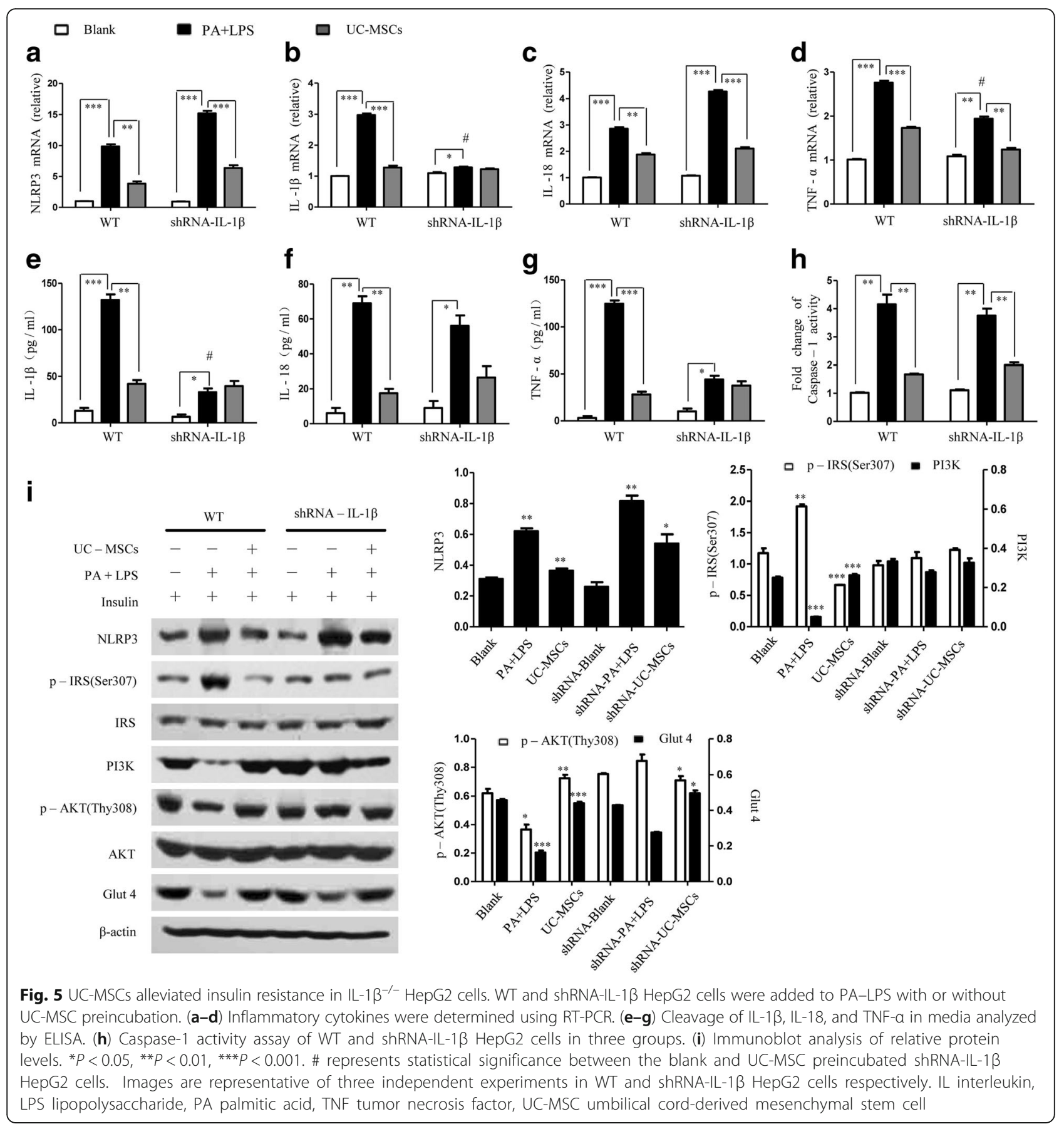

and $\mathrm{p}-\mathrm{Thr}^{308} \mathrm{AKT}$ were restored greatly in the MSCtreated group, and the elevated expression of PI3K and Glut4 translocation was also observed by MSCs even with no statistical significance. Moreover, the protein analysis of NLRP3 showed decreased expression in MSC-treated rats (Fig. 7c).

Next, we continued to analyze the influence of UCMSCs on other insulin target tissues. The mRNA expression of inflammatory cytokines (NLRP3, IL-1 $\beta$, IL-18, and TNF- $\alpha$ ) in adipose tissue was lower after MSC treatment than for T2D rats (Fig. 8a). The elevated tissue concentrations of these factors were comparable between the HFD and T2D groups, but they were also reversed by injection of MSCs (Fig. 8b). In parallel, western blot assessment revealed a decreased expression of NLRP3 in the MSC group (Fig. 8c). Similarly, as shown in muscle tissue, HFD/ STZ induced a significant increase of inflammatory factors both in gene level and protein analysis; however, this inflammation action could be partially suppressed by MSC infusion (Additional file 5: Figure S5). 


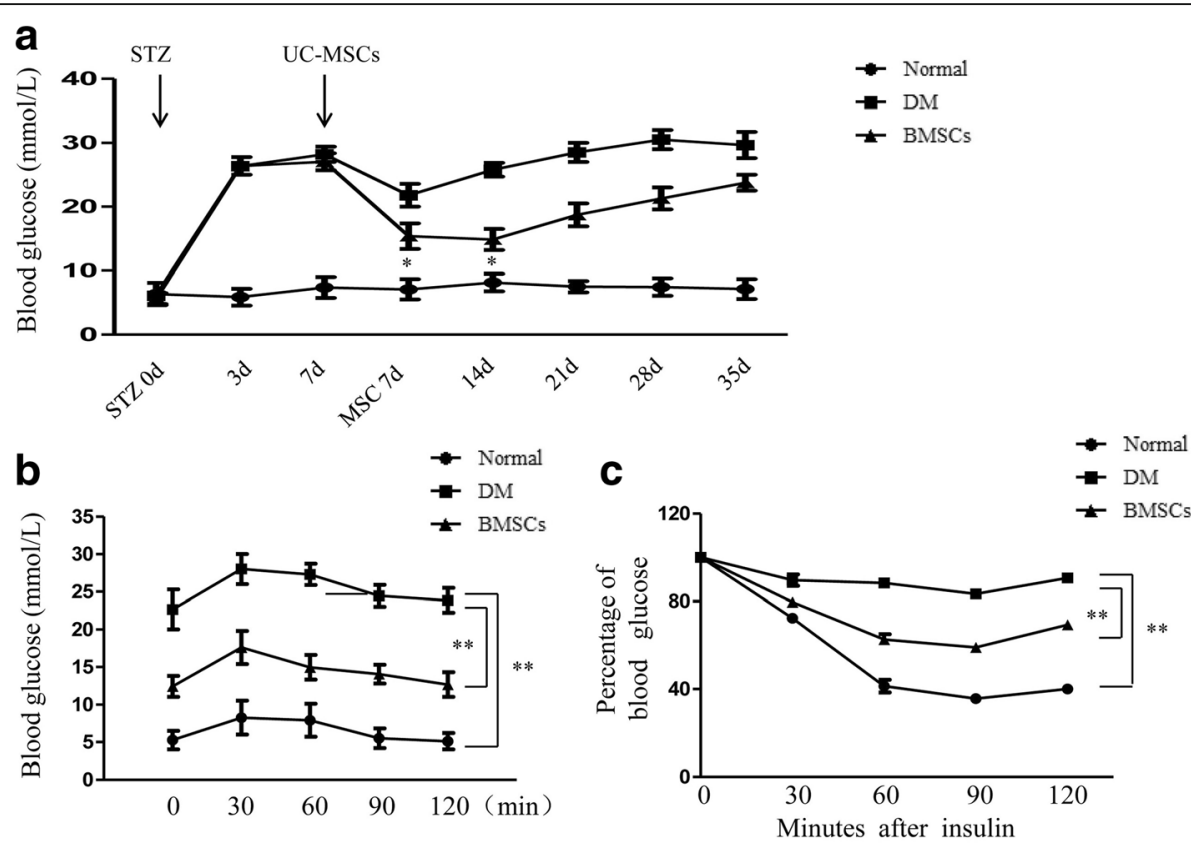

Fig. 6 UC-MSC infusion improved hyperglycemia and insulin sensitivity in T2D rats. (a) Blood glucose levels determined by a glucometer ACCUCHEK Advantage Meter in normal, diabetes mellitus (DM), and BMSC groups. (b) Glucose tolerance determined by the glucose concentration at different times. (c) Insulin tolerance assessed by IPGTT. Values are mean \pm standard deviation; $n=8$ rats each group. ${ }^{*} P<0.05,{ }^{* *} P<0.01$. UC-MSC umbilical cord-derived mesenchymal stem cell

In summary, these in-vivo experiments indicated that UC-MSCs played an anti-inflammatory role in T2D rats and effectively improved the sensitivity of peripheral target tissues to insulin.

\section{Discussion}

Insulin resistance is believed to be an early defect in $\mathrm{T} 2 \mathrm{D}$ and to play a critical role through the progression from pre T2D to eventual T2D. Despite multiple drugs having been made to ameliorate insulin resistance, we still cannot ignore the limitations of accompanying side effects. Therefore, it is urgent to search for a more effective method to improve insulin resistance. Recently, attention has been focused on the link between chronic inflammation and insulin resistance [33]. The NLRP3 inflammasome and the release of inflammatory cytokines have been indicated to cause insulin resistance, and antiinflammation therapeutic strategy is becoming promising in the treatment of T2D. Consistent with prior study, we observed inflammasome activation in an insulin resistance model of HepG2 cells, and MSC infusion not only improved insulin resistance but also impaired the activity of NLRP3 inflammation, together with a reduced caspase- 1 activity and lower expression of IL-1 $\beta$, IL-18, and TNF- $\alpha$. Studies also showed that deletion of the genes which mediated NLRP3 and IL-1 $\beta$ improved glucose utilization and increased insulin sensitivity. In vivo, MSCs inhibited NLRP3 inflammasome activation and effectively promoted insulin action by stimulating the insulin receptor signaling pathway in target tissues. Hence, we concluded that treating undesired inflammation has emerged as an attractive potential therapy for insulin-resistant T2D.

MSCs have been widely explored for their multipotent differentiation capacities and nonimmunogenicity by low expression of antigen-presenting molecules [34, 35]. Some studies have further shown that systemically infused MSCs are blocked in the lung and short-lived; no viable MSCs are found in other organs [36, 37]. More interestingly, some researchers are beginning to favor the view that MSCs exert immunomodulation and antiinflammatory effects via different paracrine mechanisms $[38,39]$. Some groups had reported that mice with rheumatoid arthritis receiving MSCs were less likely to show signs of joint inflammation than those not receiving MSCs by decreasing proinflammatory cytokines such as TNF- $\alpha$ and IL-6 [40, 41]. Moreover, therapies in MSC-treated animal models of inflammatory bowel disease demonstrated an improved survival ratio by a reduction in T cells secreting inflammatory cytokines [42]. In our experiment, we observed that MSCs cocultured with insulin-resistant HepG2 cells could reduce inflammatory action and glucose intolerance; subsequent MSC-CM treatment further certified the antiinflammatory effect of MSCs by their paracrine role. However, explicit analysis of factors released by MSCs 


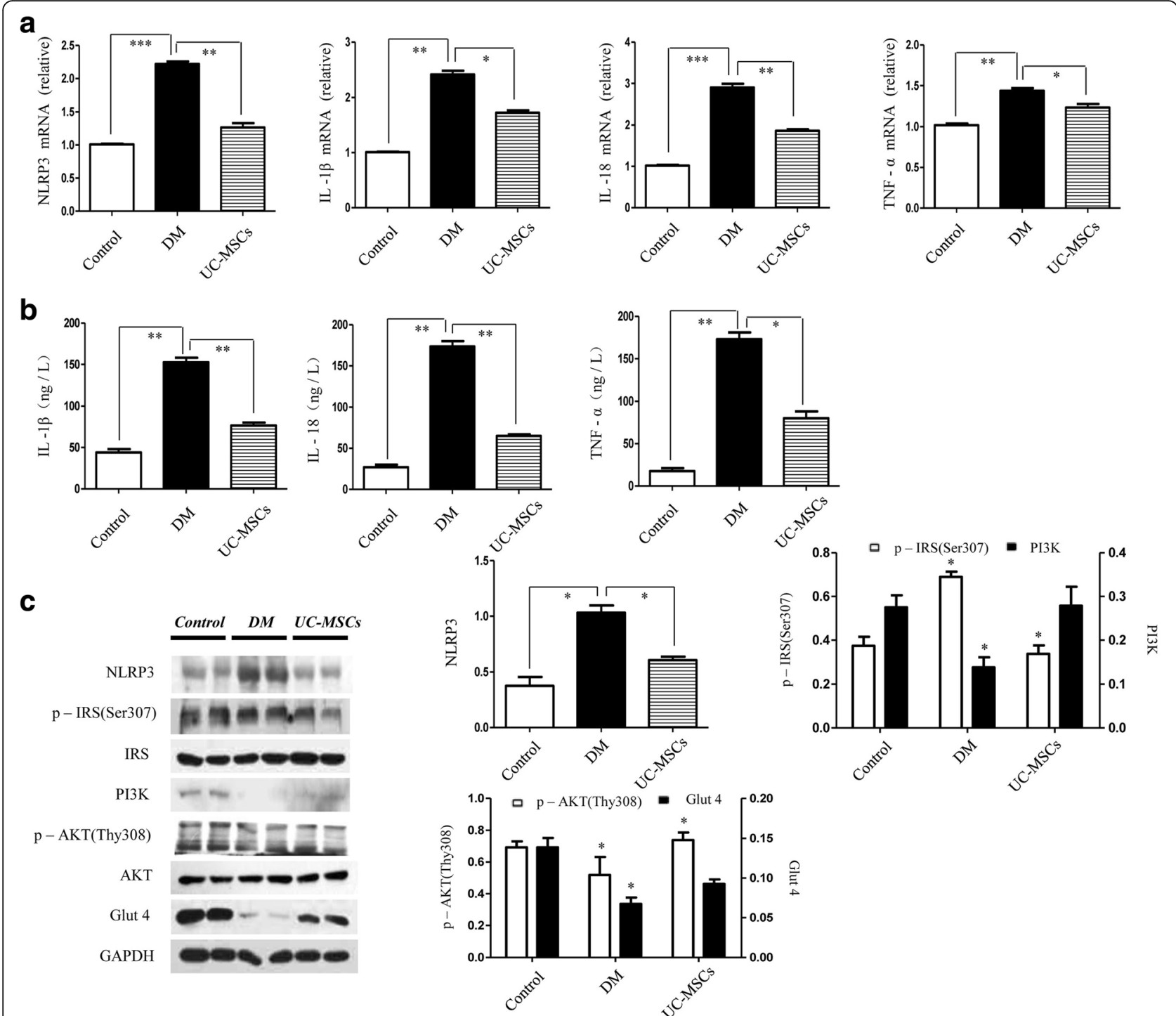

Fig. 7 UC-MSCs attenuated HFD/STZ-induced insulin resistance and inflammation in the liver. (a) Gene expression of molecules involved in the inflammation of liver; results presented relative to the control WT rats. (b) Levels of IL-1 $\beta$, IL-18, and TNF-a in the liver of all rat groups. (c) Immunoblotting analysis of liver in control WT rats, T2D rats, or MSC-treated rats. Relative protein levels of NLRP3, p-IRS, PI3K, p-AKT, and Glut4 are shown. ${ }^{*} P<0.05,{ }^{* *} P<$ $0.01,{ }^{* * *} P<0.001$. DM diabetes mellitus, IL interleukin, TNF tumor necrosis factor, UC-MSC umbilical cord-derived mesenchymal stem cell

that modulate immune responses and inflammatory reactions in alleviating insulin resistance still deserve to be explored.

In our study, we found that the inflammasome is coupled with the development of insulin resistance and severity of T2D. Therefore, we focused on exploring the role and mechanism of the NLRP3 inflammasome activation in insulin resistance. NLRP3 along with ASC and procaspase- 1 formed the inflammasome, which mediated the mature of caspase- 1 and caused the cleavage and secretion of IL- $1 \beta$ and IL-18 $[11,12]$. The processing requires two signals: the first can be achieved by LPS stimulation to induce the IL-1 $\beta$ and IL-18 production from pro-IL-1 $\beta$ and pro-IL-18; and saturated fatty acids are the second signal to activate the inflammasome to further cause the release of procaspase- 1 to caspase-1. It is well recognized that free fatty acids are substantially elevated in T2D, which become the danger signals to engage NLRP receptors and induce inflammatory factor production $[43,44]$, so we constructed the inflammation model by exposing HepG2 cells to LPS and then to PA. We also showed that PA-LPS treatment induced the inflammasome activation and the release of IL-1 $\beta$, IL-18, and TNF- $\alpha$, which could block the insulin signaling pathway. Moreover, with the lack of NLRP3 and IL-1 $\beta$, our findings revealed the improved insulin 


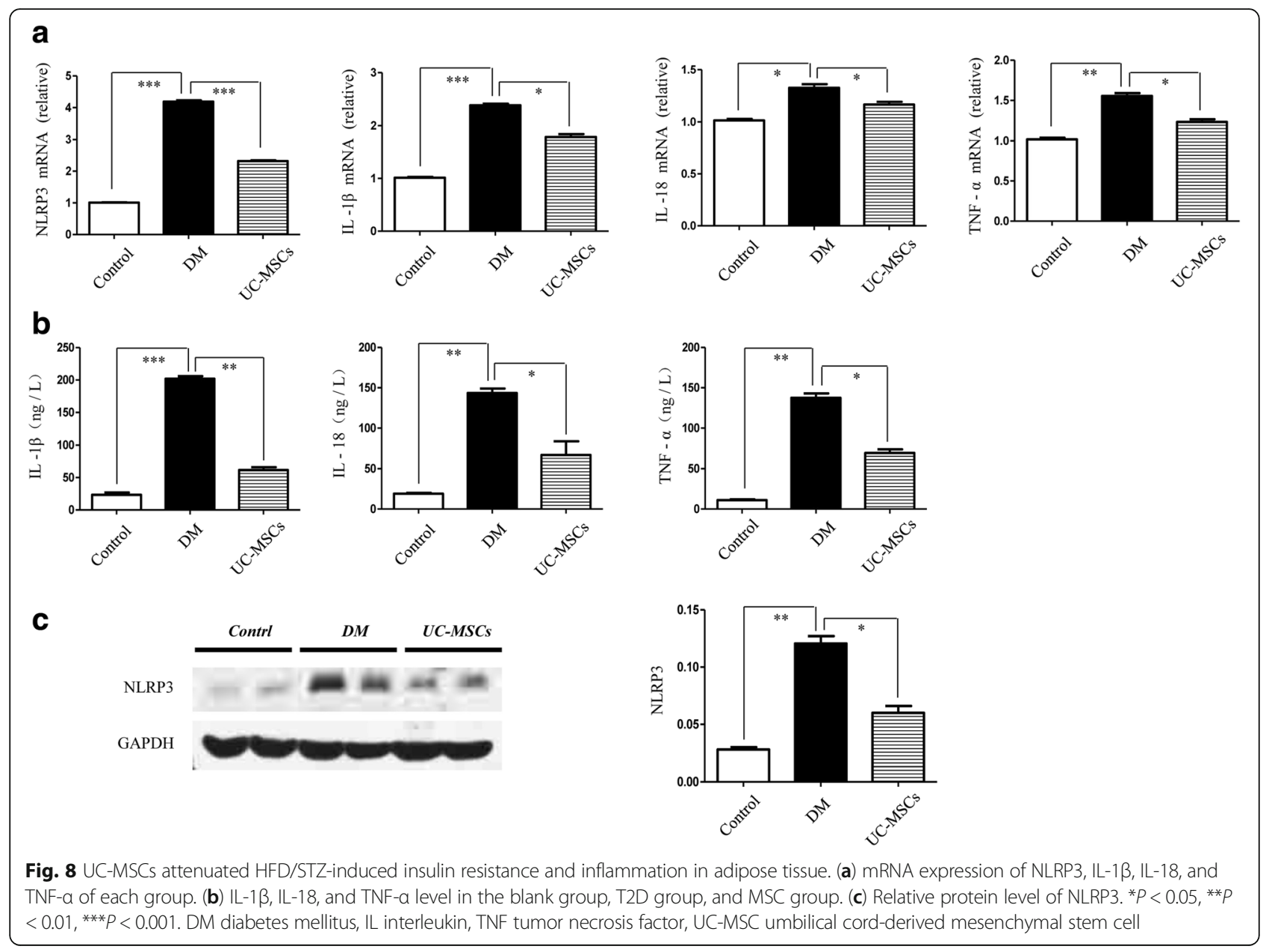

signaling cascade in vitro, suggesting the NLRP3 inflammasome as an important determiner of insulin resistance. Together these data indicated that the NLRP3 inflammasome-sensing pathway contributes to inflammation in insulin resistance. However, more studies are needed both in T2D rats and humans.

Inflammasome-mediated inflammatory cytokines were recently reported to impair insulin sensitivity [19]. Here we analyze the detrimental effects of inflammation on insulin sensitive tissues (liver, adipose, muscle). As the liver is an insulin-sensitive organ and hepatic insulin resistance precedes peripheral insulin resistance [40], HepG2 cells were chosen as the insulin resistance model in vitro. It had been established that insulin binds to its receptor to trigger a series of insulin signaling transduction pathways. The IRSPI3K-Akt pathway plays an important role in insulin's metabolic effects. Ser-307 phosphorylation of IRS-1 is considered an important negative indicator of insulin resistance, followed by reduced PI3K/Akt phosphorylation [45]. PI3K/Akt phosphorylates and further increases the Glut4 protein content in insulin target tissues. The translocations of Glut4 to cell membranes of target tissues are responsible for improvement in sensitivity to insulin action [46]. In our study, PA-LPS-induced inflammatory factors could increase the serine phosphorylation of IRS-1, resulting in decreased phosphorylation of PI3K-Akt activation and Glut4 translocation, and MSC treatment could enhance the insulin-stimulated IRS-PI3K-Akt pathway. However, LY294002, the PI3K inhibitor, prevented p-Akt activation and eventually reduced insulin sensitivity. So, we further identified that inflammation played a role in impairing the IRS-PI3K-Akt insulin signaling pathway upon PA-LPS challenge. In vivo, we further confirmed the anti-inflammatory function of MSCs in T2D rats. NLRP3-related inflammation was observed in three key insulin-sensitive organs, and MSCs were able to decrease these inflammatory mediators and recover insulin signaling transduction. These results support the concept that the anti-inflammatory action of MSCs is virtually responsible for improving insulin resistance in target tissues of T2D rats. However, we also observed that infusion of MSCs in T2D rats was able to partially promote beta-cell 
function, which might be correlated with tissue repair or cytoprotective properties of MSCs. Based on these findings, one of our future directions is to research the mechanism by which MSCs ameliorate hyperglycemia in T2D and to enhance their beneficial effects.

\section{Conclusions}

Taken together, our data indicate that stimulation of PALPS can impair glucose uptake, and evoke the inflammatory response characterized by overexpression of NLRP3-related proinflammatory cytokines, which further impair the IRSPI3K-Akt signaling pathway and eventually lead to insulin resistance in HepG2 cells. MSCs effectively inhibit NLRP3 inflammasome activation and decrease these inflammatory cytokines, contributing to the amelioration of insulin resistance. In addition, MSCs also improve inflammation-related glucose intolerance in T2D rats, further certifying their beneficial role in regulating insulin sensitivity in vivo. Therefore, these results bring a new insight into the use of MSCs' anti-inflammatory activity as another potent anti-diabetic therapy in the clinic.

\section{Additional files}

Additional file 1: Figure S1. presenting the primer sequences of NLRP3 and IL-1 $\beta$ in control-shRNA cells and shRNA cells. (JPG $146 \mathrm{~kb}$ )

Additional file 2: Figure S2. showing characterization of UC-MSCs. (a) Spindle-shaped appearance observed in UC-MSC cell colonies after passage 3 under microscopy (scale bar $=500 \mu \mathrm{m}$ ). (b, c) Multipotential capacity of UC-MSCs showing that UC-MSCs differentiated into adipocytes with lipid vesicles in the cells (scale bar $=100 \mu \mathrm{m}$ ) and osteoblasts (scale bar $=50 \mu \mathrm{m}$ ). (d) Analysis of the expression of UC-MSC surface markers by flow cytometry for mesenchymal antigens. (JPG $950 \mathrm{~kb}$ )

Additional file 3: Figure S3. showing generation of HepG2 cell models with specific NLRP3 knockdown (NLRP3 ${ }^{-1-}$ ) and IL-1 $\beta$ knockdown (IL-1 ${ }^{-1}$ -). (a) mRNA of NLRP3 and IL-1 $\beta$ examined by RT-PCR. (b) Protein levels of NLRP3 and IL-1 $\beta$ assessed by immunoblotting. Data shown as mean \pm standard deviation $(n=5)$. ${ }^{*} P<0.05$. (JPG $247 \mathrm{~kb}$ )

Additional file 4: Figure S4. showng the variation trends of serum insulin (a) and serum C-peptide (b) in control, T2D, and MSCs rats. (JPG $128 \mathrm{~kb})$

Additional file 5: Figure S5 showing UC-MSCs attenuated HFD/STZinduced insulin resistance and inflammation in muscle tissue. (a) mRNA expression of NLRP3, IL-1 $3, \mathrm{IL}-18$, and TNF-a of each group. (b) IL-1 $\beta, I L-18$, and TNF-a levels in blank, T2D, and MSC groups. (c) Relative protein level of NLRP3 by WT. ${ }^{*} P<0.05,{ }^{* *} P<0.01,{ }^{* * *} P<$ 0.001. (JPG $345 \mathrm{~kb}$ )

\section{Abbreviations}

ASC: Apoptosis-associated speck-like protein; BCA: Bicinchoninic acid; BSA: Bovine serum albumin; CM: Conditioned media; DMEM: Dulbecco's modified Eagle's medium; FBS: Fetal bovine serum; HFD: High fat diet; IL: Interleukin; LPS: Lipopolysaccharide; MSC: Mesenchymal stem cell; NLRP3: Nod-like receptor protein 3; PA: Palmitic acid; PBS: Polybutylene succinate; SD: Sprague-Dawley; STZ: Streptozotocin; T2D: Type 2 diabetes; TNF-a: Tumor necrosis factor alpha; UC-MSC: Umbilical cord-derived MSC

\section{Acknowledgements}

The authors sincerely acknowledge technical assistance from Qingwang Han and Jiejie Liu. They also thank members of Yiming Mu and Weidong Han for all of their discussion over the experiment.

\section{Funding}

This research was financially supported by the National Basic Science and Development Program (2012CB518103) and the 863 Projects of Ministry of Science and Technology of China (2012AA020502 and 2013AA020105)

\section{Availability of data and materials}

The data used or generated during the current study are included in this published article and its supplementary information files.

\section{Authors' contributions}

$X Y S, H J H, W D H$, and $Y M M$ conceived and designed the experiment. XYS, QWH, and XYS performed the experiments. XYS and HJH analyzed the data. $\mathrm{QWH}, J J L, L D$, and YMM contributed reagents/materials/analysis tools. XYS, $\mathrm{HJH}$, and YMM wrote the paper. All authors read and approved the final manuscript.

\section{Ethics approval and consent to participate}

The animal-related experiments were conducted under the guidelines and approved by the Ethic Committee of the Chinese PLA General Hospital (Reference number: 2013022177). Human umbilical cord tissue was collected from healthy women after deliveries in the Chinese PLA General Hospital, and isolation and purification with the approval of ethic Committee of the Chinese PLA General Hospital (Reference number: 2012020470) and with anonymous consent from the donors.

\section{Consent for publication}

Not applicable.

\section{Competing interests}

The authors declare that they have no competing interests.

\section{Publisher's Note}

Springer Nature remains neutral with regard to jurisdictional claims in published maps and institutional affiliations.

Received: 26 June 2017 Revised: 27 August 2017 Accepted: 12 September 2017 Published online: 02 November 2017

\section{References}

1. Mokdad AH, Ford ES, Bowman BA, Dietz WH, Vinicor F, et al. Prevalence of obesity, diabetes, and obesity-related health risk factors. JAMA. 2003; 289(1):76-9.

2. Reaven GM. Role of insulin resistance in human diseases. Diabetes. 1988;37: 1595-607.

3. Spranger J, Kroke A, Mohlig M, Hoffmann K, Bergmann MM, et al. Inflammatory cytokines and the risk to develop type 2 diabetes: results of the prospective population-based European Prospective Investigation into Cancer and Nutrition (EPIC)—Potsdam Study. Diabetes. 2003;52(3):812-7.

4. Wellen KE, Hotamisligil GS. Inflammation, stress, and diabetes. J Clin Invest. 2005;115:1111-9.

5. Hotamisligil GS, Shargill NS, Spiegelman BM. Adipose expression of tumor necrosis factor-alpha: direct role in obesity-linked insulin resistance. Science. 1993;259:87-91.

6. Visser M, Bouter LM, McQuillan GM, Wener MH, Harris TB. Elevated C-reactive protein levels in overweight and obese adults. JAMA. 1999;282:2131-5.

7. Stienstra R, Tack CJ, Kanneganti TD, Joosten LA, Netea MG. The inflammasome puts obesity in the danger zone. Cell Metab. 2012;15(1):10-8.

8. Wen H, Gris D, Lei Y, Jha S, Zhang L, et al. Fatty acid-induced NLRP3-ASC inflammasome activation interferes with insulin signaling. Nat Immunol. 2011;12:208-15.

9. Martinon F. Detection of immune danger signals by NALP3. J Leukoc Biol. 2008:83:507-11.

10. Petrilli V, Dostert C, Muruve DA, Tschopp J. The inflammasome: a danger sensing complex triggering innate immunity. Curr Opin Immunol. 2007;19:615-22. 
11. Ting JP, Willingham SB, Bergstralh DT. NLRs at the intersection of cell death and immunity. Nat Rev Immunol. 2008;8:372-9.

12. Schroder K, Tschopp J. The inflammasomes. Cell. 2010;140:821-32.

13. Lee HM, Kim JJ, Kim HJ, Shong M, Ku BJ, et al. Upregulated NLRP3 inflammasome activation in patients with type 2 diabetes. Diabetes. 2013; 62(1):194-204.

14. De Nardo D, Latz E. NLRP3 Inflammasomes link inflammation and metabolic disease. Trends Immunol. 2011:32:373-9.

15. Stienstra R, van Diepen JA, Tack CJ, Zaki MH, van de Veerdonk FL, et al. Inflammasome is a central player in the induction of obesity and insulin resistance. Proc Natl Acad Sci U S A. 2011;108(37):15324-9.

16. Hotamisligil GS. Inflammation and metabolic disorders. Nature. 2006;444:860-7.

17. Leinonen E, Hurt-Camejo E, Wiklund O, Hulten LM, Hiukka A, et al. Insulin resistance and adiposity correlate with acute-phase reaction and soluble cell adhesion molecules in type 2 diabetes. Atherosclerosis. 2003;166:387-94.

18. Maedler K, Dharmadhikari G, Schumann DM, Størling J. Interleukin-1 beta targeted therapy for type 2 diabetes. Expert Opin Biol Ther. 2009;9(9):1177-88.

19. Stienstra R, Joosten LA, Koenen T, van Tits B, van Diepen JA, et al. The inflammasome-mediated caspase- 1 activation controls adipocyte differentiation and insulin sensitivity. Cell Metab. 2010;12(6):593-605.

20. Vandanmagsar B, Youm YH, Ravussin A, Galgani JE, Stadler K, et al. The NLRP3 inflammasome instigates obesity-induced inflammation and insulin resistance. Nat Med. 2011;17:179-88.

21. Si YL, Zhao YL, Hao HJ, Fu XB, Han WD. MSCs: biological characteristics, clinical applications and their outstanding concerns. Ageing Res Rev. 2011;10:93-103.

22. Baraniak PR, McDevitt TC. Stem cell paracrine actions and tissue regeneration. Regen Med. 2010;5(1):121-43.

23. Zhu Q, Li XX, Wang W, Hu J, Li PL, et al. Mesenchymal stem cell transplantation inhibited high salt-induced activation of the NLRP3 inflammasome in the renal medulla in Dahl S rats. Am J Physiol Renal Physiol. 2016;310(7):F621-F627.

24. Manferdini C, Maumus M, Gabusi E, Piacentini A, Filardo G, et al. Adiposederived mesenchymal stem cells exert antiinflammatory effects on chondrocytes and synoviocytes from osteoarthritis patients through prostaglandin E2. Arthritis Rheum. 2013;65(5):1271-81.

25. Liu X, Zheng P, Wang X, Dai G, Cheng $H$, et al. A preliminary evaluation of efficacy and safety of Wharton's jelly mesenchymal stem cell transplantation in patients with type 2 diabetes mellitus. Stem Cell Res Ther. 2014;5(2):57.

26. Si Y, Zhao Y, Hao H, Liu J, Guo Y, et al. Infusion of mesenchymal stem cells ameliorates hyperglycemia in type 2 diabetic rats: identification of a novel role in improving insulin sensitivity. Diabetes. 2012;61:1616-25.

27. Mageed AS, Pietryga DW, DeHeer DH, West RA. Isolation of large numbers of mesenchymal stem cells from the washings of bone marrow collection bags: characterization of fresh mesenchymal stem cells. Transplantation. 2007:83:1019-26.

28. Bieback K, Kern S, Klüter $\mathrm{H}$, Eichler $\mathrm{H}$. Critical parameters for the isolation of mesenchymal stem cells from umbilical cord blood. Stem Cells. 2004;22:625-34

29. Xie Z, Hao H, Tong $C$, Cheng Y, Liu J, et al. Human umbilical cord-derived mesenchymal stem cells elicit macrophages into an anti-inflammatory phenotype to alleviate insulin resistance in type 2 diabetic rats. Stem Cells 2016:34(3):627-39.

30. Pan XH, Zhu L, Yao X, Liu JF, Li ZA, et al. Development of a tree shrew metabolic syndrome model and use of umbilical cord mesenchymal stem cell transplantation for treatment. Cytotechnology. 2016;68(6):2449-67.

31. Maranda EL, Rodriquez-Menocal L, Badiavas EV. Role of mesenchymal stem cells in dermal repair in burns and diabetic wounds. Curr Stem Cell Res Ther. 2017;12(1):61-70

32. Ando $Y$, Matsubara $K$, Ishikawa J, Fujio M, Shohara R, et al. Stem cellconditioned medium accelerates distraction osteogenesis through multiple regenerative mechanisms. Bone. 2014;61:82-90.

33. Nieto-Vazquez I, Fernandez-Veledo S, Kramer DK, Vila-Bedmar R, GarciaGuerral L, et al. Insulin resistance associated to obesity: the link TNF-alpha. Arch Physiol Biochem. 2008;114(3):183-94.

34. Pittenger MF, Mackay AM, Beck SC, Jaiswal RK, Douglas R, et al. Multilineage potential of adult human mesenchymal stem cells. Science. 1999:284:143-7.

35. Prockop DJ. Repair of tissues by adult stem/progenitor cells (MSCs): controversies, myths, and changing paradigms. Mol Ther. 2009;17:939-46.

36. Eggenhofer E, Benseler V, Kroemer A, Popp FC, Geissler EK, et al. Mesenchymal stem cells are short-lived and do not migrate beyond the lungs after intravenous infusion. Front Immunol. 2012;3:297.
37. Lee RH, Pulin AA, Seo MJ, Kota DJ, Ylostalo J, et al. Intravenous hMSCs improve myocardial infarction in mice because cells embolized in lung are activated to secrete the anti-inflammatory protein TSG-6. Cell Stem Cell. 2009;5:54-63.

38. Prockop DJ, Oh JY. Mesenchymal stem/stromal cells (MSCs) role as guardians of inflammation. Mol Ther. 2012;20:14-20.

39. Madrigal M, Rao KS, Riordan NH. A review of therapeutic effects of mesenchymal stem cell secretions and induction of secretory modification by different culture methods. J Transl Med. 2014;12:260.

40. González MA, Gonzalez-Rey E, Rico L, Büscher D, Delgado M. Treatment of experimental arthritis by inducing immune tolerance with human adiposederived mesenchymal stem cells. Arithrtis Rheum. 2009;60:1006-19.

41. Liu Y, Mu R, Wang S, Long L, Liu X, et al. Therapeutic potential of human umbilical cord mesenchymal stem cells in the treatment of rheumatoid arthritis. Arthritis Res Ther. 2010:12:R210.

42. Zhang Q, Shi S, Liu Y, Uyanne J, Shi Y, et al. Mesenchymal stem cells derived from human ginggiva are capable of immunomodulatory functions and ameliorate inflammation-related tissue destruction in experimental colitis. J Immunol. 2009;183:7787-98.

43. Boden $\mathrm{G}$. Interaction between free fatty acids and glucose metabolism. Curr Opin Clin. 2002:5:545-9.

44. Shi H, Kokoeva MV, Inouye K, Tzameli I, Yin H, et al. TLR4 links innate immunity and fatty acid-induced insulin resistance. J Clin Invest. 2006; 116(11):3015-25.

45. Gual P, Le Marchand-Brustel Y, Tanti JF. Positive and negative regulation of insulin signaling through IRS-1 phosphorylation. Biochimie. 2005:87:99-109.

46. Zisman A, Peroni OD, Abel ED, Michael MD, Mauvais-Jarvis F, et al. Targeted disruption of the glucose transporter 4 selectively in muscle causes insulin resistance and glucose intolerance. Nat Med. 2000;6:924-8.

\section{Submit your next manuscript to BioMed Central and we will help you at every step:}

- We accept pre-submission inquiries

- Our selector tool helps you to find the most relevant journal

- We provide round the clock customer support

- Convenient online submission

- Thorough peer review

- Inclusion in PubMed and all major indexing services

- Maximum visibility for your research

Submit your manuscript at www.biomedcentral.com/submit
) Biomed Central 